\title{
Dataset: Thermal Energy Harvesting Profiles in Residential Settings
}

\author{
Victor Ariel Leal Sobral \\ University of Virginia \\ Charlottesville, USA \\ sobral@virginia.edu \\ Jonathan L. Goodall \\ University of Virginia \\ Charlottesville, USA \\ goodall@virginia.edu
}

\author{
John Lach \\ The George Washington University \\ Washington DC, USA \\ jlach@gwu.edu \\ Bradford Campbell \\ University of Virginia \\ Charlottesville, USA \\ bradjc@virginia.edu
}

\begin{abstract}
While relying on energy harvesting to power Internet of Things (IoT) devices eliminates the maintenance burden of battery replacement, energy generation fluctuation constitutes a major source of uncertainty to design reliable self-powered IoT devices. To characterize spatial-temporal variability of energy harvesting, data acquisition campaigns are needed across the range of potential harvesting sources. In this work we present a dataset to characterize thermal energy sources in residential settings by measuring thermoelectric generator (TEG) operating conditions over 16 deployment locations for periods ranging from 19 to 53 days. We present our easy-touse thermal energy measurement platform built from off-the-shelf component modules and a custom TEG interface circuit. We demonstrate how the collected measurements can inform the design of energy harvesting IoT devices by deriving the TEG's maximum power output and estimating the available energy at each harvesting location.
\end{abstract}

\section{CCS CONCEPTS}

- Hardware $\rightarrow$ Sensors and actuators; Sensor applications and deployments; Sensor devices and platforms; Renewable energy.

\section{KEYWORDS}

Energy Harvesting, Dataset, Residential Settings, Thermoelectric Generator, Internet of Things

\section{ACM Reference Format:}

Victor Ariel Leal Sobral, John Lach, Jonathan L. Goodall, and Bradford Campbell. 2021. Dataset: Thermal Energy Harvesting Profiles in Residential Settings. In The 19th ACM Conference on Embedded Networked Sensor Systems (SenSys '21), November 15-17, 2021, Coimbra, Portugal. ACM, New York, NY, USA, 4 pages. https://doi.org/10.1145/3485730.3494111

Permission to make digital or hard copies of all or part of this work for personal or classroom use is granted without fee provided that copies are not made or distributed for profit or commercial advantage and that copies bear this notice and the full citation on the first page. Copyrights for components of this work owned by others than ACM must be honored. Abstracting with credit is permitted. To copy otherwise, or republish, to post on servers or to redistribute to lists, requires prior specific permission and/or a fee. Request permissions from permissions@acm.org.

SenSys'21, November 15-17, 2021, Coimbra, Portugal

(c) 2021 Copyright is held by the owner/author(s).

ACM ISBN 978-1-4503-9097-2/21/11

https://doi.org/10.1145/3485730.3494111

\section{INTRODUCTION}

Energy harvesting is a promising solution to enable ubiquitous Internet of Things (IoT) infrastructure as it can potentially eliminate human intervention burden associated with battery replacement. However, the design of reliable energy harvesting IoT devices is challenging due to energy availability fluctuations resulting from particular environment conditions surrounding each device. A common approach to characterize spatial-temporal variability of energy harvesting conditions is to extensively collect energy harvesting traces under relevant environments and later use this information to derive harvester requirements and power consumption constraints to IoT applications. While significant efforts have been made to collect energy harvesting traces from solar harvesters [2, 5], traces from other energy sources as thermal energy are scarce. Collecting energy harvesting traces from thermal sources can support IoT designers to evaluate if thermal energy is a viable option to enable their application, either being used as the main source of energy or as a complementary source.

To address this energy harvesting knowledge gap, we present a thermal energy harvesting profiling dataset in residential settings to support energy harvesting IoT applications for home and building automation. Our measuring platform collects a thermoelectric generator (TEG) operating conditions as well as temperature readings using off-the-shelf component modules and a custom circuit module to interface with the TEG. As part of an IRB approved study, our platform was deployed by volunteers on 16 different locations including pipes carrying warm water, electronic devices, a refrigerator compressor, and an HVAC vent. The data collection period varied for each location, the shortest deployment lasting 19 days and the longest 53 days. In total, the dataset contains 544 days worth of thermal energy recordings. Using the collected measurements, we estimated the TEG's internal resistance, then computed the TEG's maximum power point (MPP) and simulated the output current of a LTC3108 boost converter circuit. To the best of our knowledge this is the first study to spatially and temporally characterize thermal energy sources in residential settings for multiple weeks. The dataset and python scripts to process and reproduce plots are available in [4] through LibraData, the University of Virginia's data repository ( https://doi.org/10.18130/V3/M9CP9C). 


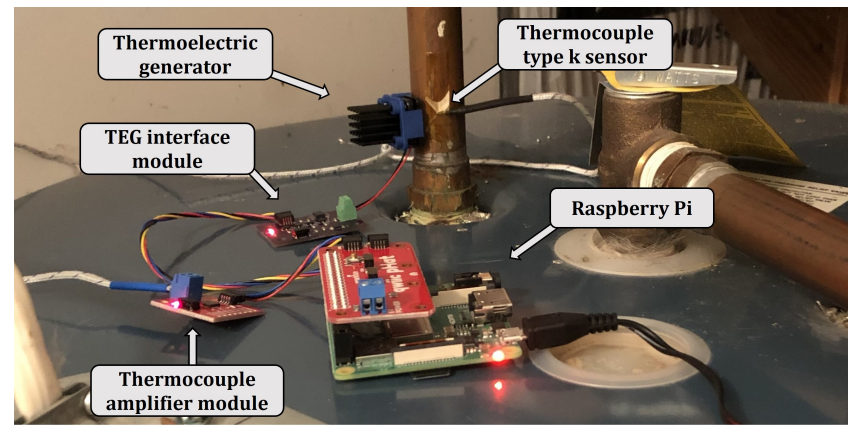

Figure 1: Thermal energy harvesting recording platform deployed at a water boiler location (dataset: TEG001_env1.h5).

\section{THERMAL ENERGY HARVESTING RECORDING PLATFORM}

The energy harvesting recording platform used for this study follows the I-V (current-voltage) curve measurement approach introduced by [3], consisting on measuring the energy harvester output under a range of loading conditions. The advantage of using this technique is providing a realistic joint characterization of environment and harvester that is independent of specific harvester loading, allowing circuit level simulations of boost converter interfaces and the estimation of generated power available to the IoT device. The I-V curve measurements were performed by a custom circuit module consisting of an analog to digital converter (ADC) and four load channels $(0.1,0.47,1.5$ and $4.7 \mathrm{ohms})$ that can be independently switched on or off in order to measure the TEG's output voltage under each load and hence allow an estimation of the TEG I-V curve. The resistor loads were chosen to cover the TEG expected I-V curve range, considering the TEG manufacturer's recommended optimal load of $1.5 \mathrm{ohms}$. To calibrate the platform measurements, the resistance of each active channel was measured $(0.2251,0.5990,1.6245,4.8070 \mathrm{ohms}$ respectively) as well as the short-circuited probe's resistance $(0.1080 \mathrm{ohms})$. The ADC used was an ADS1015, configured to provide 12-bit voltage readings and a gain of 8 , resulting in a voltage range of $[-0.512 \mathrm{~V},+0.512 \mathrm{~V}]$ and resolution of $0.25 \mathrm{mV}$. Finally, the hot surface side and ambient temperature parameters of the TEG operating conditions were also measured as additional information valuable to harvester designers.

To improve accessibility and make our investigations reproducible to the largest number of researchers and makers possible, we adopted mostly off-the-shelf component modules for our thermal energy harvesting recording platform. We use a Raspberry Pi as a computing device, a thermocouple amplifier module to acquire temperature readings and a custom module to measure the TEG output voltage under four resistor loads and open circuit conditions. This approach allows the platform to be more easily assembled while also providing flexibility to reuse parts to other projects. The thermal energy harvesting recording platform is assembled using the components listed in Table 1 and the platform is depicted in Figure 1. In Figure 2 we show a block diagram of the data acquisition platform and in Figure 3 we show circuit schematics details of the custom TEG interface module.

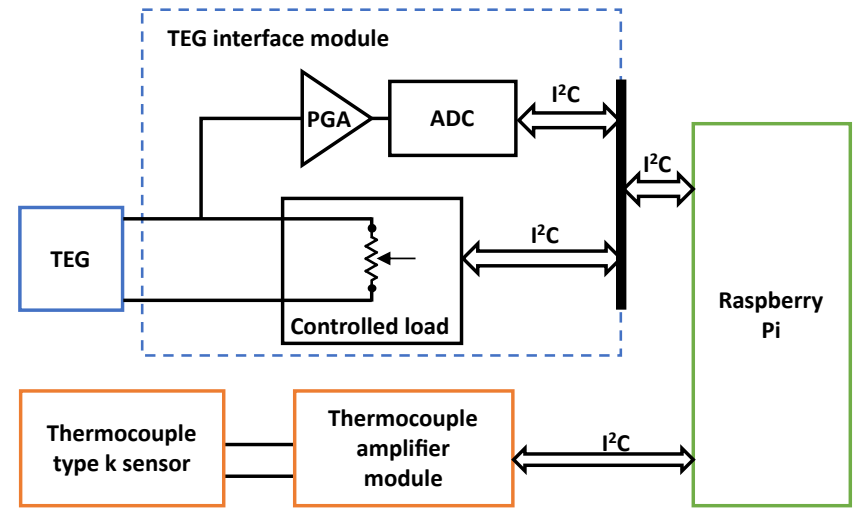

Figure 2: Block diagram of the data acquisition platform. We use a custom TEG interface module to switch the load connected to the TEG and to measure the TEG output voltage for each loading condition. We also use an off-the-shelf thermocouple amplifier module with a type $\mathrm{k}$ thermocouple sensor to measure the TEG hot side surface temperature as well as the ambient temperature. We use an Raspberry Pi as a controller and data logger, connected to both modules through an I2C interface.

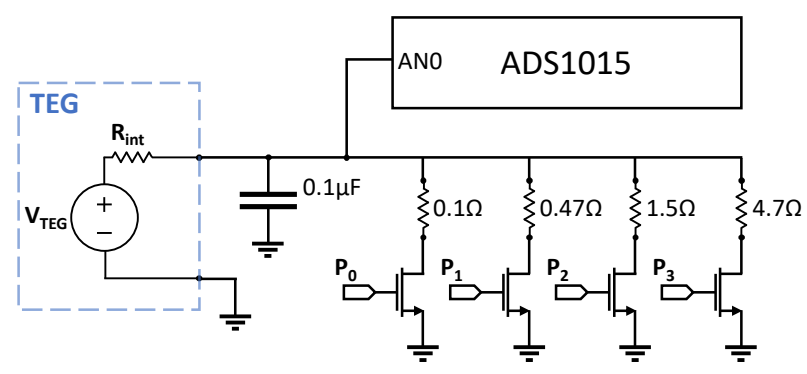

Figure 3: TEG interface module schematics. We selected four fixed resistor loads to characterize the TEG model by assuming $R_{\text {int }}$ around $1.5 \mathrm{ohms}$. We used MOSFET transistors to switch each load ON or OFF and we used an ADS1015 IC as the programmable gain amplifier (PGA) and the analog to digital converter (ADC). $P_{0}$ to $P_{3}$ are digital output ports of a PCA9536 IC, an I2C controlled GPIO. We interface the ADS1015 and the PCA9536 ICs with an Raspberry Pi controller through I2C.

\section{DATASET}

The thermal energy harvesting recording platform was configured to perform one measurement every 0.5 seconds consisting of: the TEG hot surface and ambient temperature; and the TEG output voltage under open-circuit condition as well as connected to each load resistor $(0.1,0.47,1.5$ and $4.7 \mathrm{ohms})$. Measured data points are saved as CSV files in the Raspberry Pi SD card. The sampling period of 0.5 seconds was chosen as a compromise between data set size and time domain resolution to monitor typical thermal sources present in residential settings. 
Table 1: Thermal energy harvesting recording platform components

\begin{tabular}{c} 
Component list specification \\
\hline Raspberry pi 3 model A+ with a 32GB microSDHC UHS-1 A1 card (Sandisk) and a 2.5A microUSB power supply (Pro-elec). \\
Qwiic pHat board (Sparkfun) and 2 qwiic cables to connect modules over I2C interface. \\
Qwiic Thermocouple Amplifier - MCP9600 (Sparkfun) with a K-type thermocouple (Pimoroni). \\
Custom PCB for TEG I-V curve profiling based on PCA9536 and ADS1015 integrated circuits. \\
Mini-Harvester Thermal Energy Generator (Marlow EHA-PA1AN1-R03) with 1.5 ohms optimal load and 20x20mm surface area.
\end{tabular}

Volunteers installed the energy harvesting recording platform in 16 residential locations following an approved IRB protocol and they provided a picture of each installation setup, shared as part of the dataset. Seven of the selected locations were over warm water conducting pipes close to water boilers. Five were under sinks, either over warm water conducting pipes or over wastewater conducting pipes. The following locations only had one deployment: over a WiFi router, over a NAS data storage system, attached to a heater system, and over a refrigerator's compressor. Due to practical deployment challenges of the profiling devices, accidents during the deployment resulted in disconnected TEG devices or invalid temperature readings. To help filtering data, boolean flag columns were added to the dataset to identify data points related to poor deployment conditions.

To complement the dataset, we estimated the TEG internal resistance from our measurements by performing a least squares fit of the I-V curve data points at each timestamp value. For the used TEG device under typical operating conditions, the estimated internal resistance is approximated to either 1.2 or $1.3 \mathrm{ohms}$. Using the estimated internal resistance, we calculated the maximum power point (MPP) of the TEG device and the maximum power density of this device by dividing the MPP to the TEG surface area. Finally, we interpolated the LTC 3108 curves provided in the manufacturer data sheet [1] to derive the relationship between TEG open-circuit voltage and output charging current for the estimated TEG internal resistance of either $1.2 \mathrm{ohms}$ or $1.3 \mathrm{ohms}$. This open-circuit voltage to charge current curve represents a LTC 3108 based boost converter circuit with a 1:100 ratio transformer as specified in page five of [1]. All the dataset measured and calculated parameters are summarized in Table 2. The dataset were formatted using python Pandas module as dataframes and saved as Hierarchical Data Format (HDF) files using the function "pandas.to_hdf()" with default settings.

\section{DATASET USE CASE EXAMPLES}

A straight forward dataset use case would be evaluating how much energy can be generated at different locations as a first estimate of application feasibility. For instance, the calculated TEG MPP output represents the best case scenario for the used TEG device in the recorded conditions. Figure 4 depicts power generation distribution at MPP for two boiler locations deployments as in Figure 1. An IoT designer could use these distributions to evaluate what fraction of the time the power available could support an specific application or it could also be used to determine if an application outcome is expected to be similar for these locations. As a reference, the harvesting levels of hundreds of micro-watts recorded at the boiler

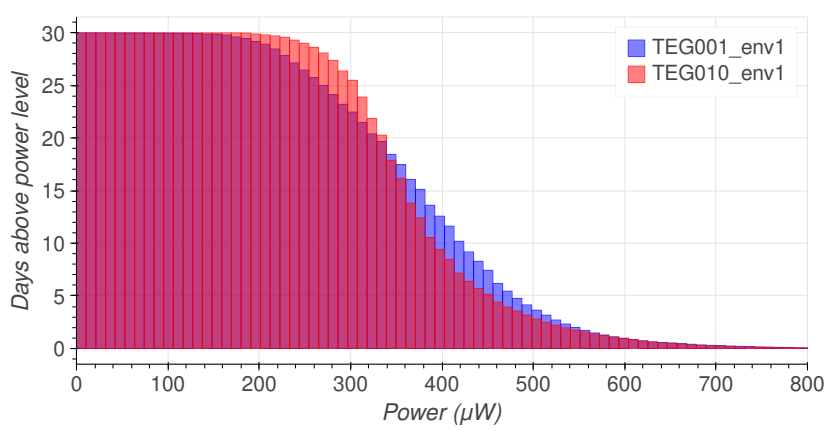

Figure 4: Both recorded environments near boilers have potential to generate $330 \mu \mathrm{W}$ or more for two thirds of the time, however environment TEG010_env1 can generate at least $260 \mu \mathrm{W}$ more consistently than TEG001_env1.

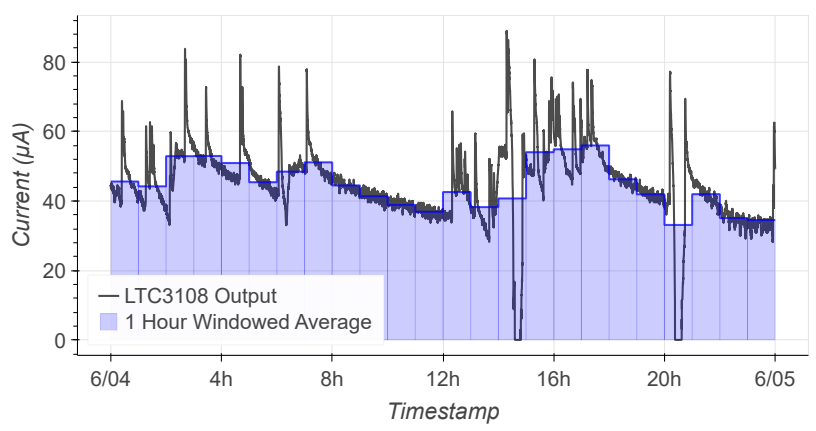

Figure 5: Using the dataset with a LTC3108 circuit model, we simulated the net current available to an IoT device. For the shown period of dataset TEG001_env1, an IoT designer can select an energy storage device to buffer energy through one hour windows and set an energy-adaptive device to operate with average current consumption modes between 32 and 56 $\mu \mathrm{A}$.

environments is comparable to the energy harvested by small solar cells in low-level indoor light conditions [6].

While the TEG MPP represents an upper bound on the available energy to an IoT device, the boost converter output current provides a more realistic estimation of the IoT's net usable energy by taking into account practical circuit efficiency parameters. Using the TEG internal resistance and the LTC3108 boost converter circuit model curves previously mentioned, it is possible to estimate the output current that is available to charge a storage device, for instance a 
Table 2: Thermal energy harvesting profiler dataset parameters

\begin{tabular}{ccc}
\hline Parameter & Unit & Description \\
\hline timestamp & milliseconds & Unix timestamp in UTC \\
voltage_open_circuit & volts & TEG open-circuit output voltage \\
voltage_R_p1 & volts & TEG output voltage with 0.1 ohm load \\
voltage_R_p47 & volts & TEG output voltage with 0.47 ohm load \\
voltage_R_1p5 & volts & TEG output voltage with 1.5 ohm load \\
voltage_R_4p7 & volts & TEG output voltage with 4.7 ohm load \\
temperature_ambient & degree Celsius & Ambient temperature \\
temperature_surface & degree Celsius & TEG hot surface temperature \\
teg_internal_resistance & ohms & Internal resistance of the TEG model \\
teg_mpp_uw & microwatts & TEG maximum power point (MPP) output \\
teg_mpp_density_uw_per_cm2 & microwatts / centimeter squared & TEG MPP density \\
boost_voc_mv & millivolts & TEG open circuit voltage input for LTC3108 model \\
boost_ichg_ua & microamperes & Charging current output of LTC3108 model \\
flag_thermocouple_invalid & - & Boolean flag for invalid temperature measurements \\
flag_teg_disconnected & - & Boolean flag for invalid TEG measurements \\
\hline
\end{tabular}

super capacitor. Figure 5 depicts the estimated charging current output profile of the LTC3108 boost converter if it was connected to the TEG in the recorded environments. This profile can inform how long it would take to a capacitor to fully charge in these conditions and what is the expected maximum average current consumption of a energy harvesting IoT device for this environment. Furthemore, energy harvesting prediction algorithms to adapt IoT operation given energy generation fluctuations can also be evaluated using this dataset as performed by [5].

\section{ACKNOWLEDGEMENTS}

This work was supported in part by the National Science Foundation under grant CRISP-1735587.

\section{REFERENCES}

[1] Analog Devices 2019. LTC3108 DC/DC converter IC (Rev D.). Analog Devices. https://www.analog.com/en/products/ltc3108.html.

[2] Maria Gorlatova, Aya Wallwater, and Gil Zussman. 2011. Networking low-power energy harvesting devices: Measurements and algorithms. In 2011 Proceedings IEEE INFOCOM. 1602-1610. https://doi.org/10.1109/INFCOM.2011.5934952

[3] Josiah Hester, Lanny Sitanayah, Timothy Scott, and Jacob Sorber. 2017. Realistic and Repeatable Emulation of Energy Harvesting Environments. ACM Trans. Sen. Netw. 13, 2, Article 16 (April 2017), 33 pages. https://doi.org/10.1145/3064839

[4] Victor Ariel Leal Sobral, John Lach, Jonathan L. Goodall, and Bradford Campbell. 2021. Thermal Energy Harvesting Profiles in Residential Settings. https://doi.org/ 10.18130/V3/M9CP9C

[5] Lukas Sigrist, Andres Gomez, and Lothar Thiele. 2019. Dataset: Tracing Indoor Solar Harvesting. In Proceedings of the 2nd Workshop on Data Acquisition To Analysis (New York, NY, USA) (DATA'19). Association for Computing Machinery, New York, NY, USA, 47-50. https://doi.org/10.1145/3359427.3361910

[6] Alexander J. Williams, Matheus F. Torquato, Ian M. Cameron, Ashraf A. Fahmy, and Johann Sienz. 2021. Survey of Energy Harvesting Technologies for Wireless Sensor Networks. IEEE Access 9 (2021), 77493-77510. https://doi.org/10.1109/ ACCESS.2021.3083697 